\title{
Is There an Association between Temperament and Apolipoprotein E? A Replication of a 1993 Young Finns Study
}

\author{
Aino M. Pitkänen ${ }^{1}$, Päivi Merjonen ${ }^{1}$ Liisa Keltikangas-Järvinen ${ }^{1}$ Ilkka Seppälä², \\ Terho Lehtimäki ${ }^{2}$, Jorma Viikari ${ }^{3}$, Olli T. Raitakari ${ }^{4}$, Mirka Hintsanen ${ }^{1,5^{*}}$ \\ ${ }^{1}$ Institute of Behavioural Sciences, University of Helsinki, Helsinki, Finland \\ ${ }^{2}$ Department of Clinical Chemistry, Fimlab Laboratories, School of Medicine, \\ University of Tampere, Tampere University Hospital, Tampere, Finland \\ ${ }^{3}$ Department of Medicine, University of Turku, Turku University Hospital, Turku, Finland \\ ${ }^{4}$ Research Centre of Applied and Preventive Cardiovascular Medicine, Department of Clinical Physiology and Nuclear Medicine, \\ University of Turku, Turku University Hospital, Turku, Finland \\ ${ }^{5}$ Helsinki Collegium for Advanced Studies, University of Helsinki, Helsinki, Finland \\ Email: ${ }^{*}$ mirka.hintsanen@helsinki.fi
}

Received January 21, 2013; revised March 21, 2013; accepted May 6, 2013

Copyright (C) 2013 Aino M. Pitkänen et al. This is an open access article distributed under the Creative Commons Attribution License, which permits unrestricted use, distribution, and reproduction in any medium, provided the original work is properly cited.

\begin{abstract}
Background: An association between apolipoprotein $E(a p o E)$ gene polymorphism and temperament has been found in the Young Finns cohort. Motor activity in childhood and mental vitality, sociability and positive emotionality in adolescence were associated with $a p o E$. Two research groups have attempted to replicate these findings but no associations have been found. Purpose: The purpose of the present study was to confirm the original findings with new and more reliable genotyping from a larger sample derived from the same Young Finns Study as the original finding. Methods: The study included 2808 participants aged 3 - 18 years in 1980. The same methods in assessing temperament were used as in the original study. Temperament was operationalized as motor activity, cooperativeness, negative emotionality, mental vitality, sociability and positive emotionality. Temperament was assessed by participants' mothers in 1980 and 1983 and self-rated in 1983 by adolescent participants. Results: Motor activity was not associated with apoE polymerphisms. All other previous results were replicated. Adolescents' positive emotionality, mental vitality and sociability were associated with $a p o E$. Conclusions: The results indicated that there is an association between temperament and $a p o E$. The previous absence of association between temperament and $a p o E$ in the replication studies may be due to the fact that researchers used different dimensions of temperament and thus probably studied different phenomena. Cultural differences in personality assessment might also explain the contradictory findings.
\end{abstract}

Keywords: Temperament; Personality; Behavioral Genetics; Biological Psychology; apoE

\section{Introduction}

Apolipoprotein $E(a p o E)$ is a gene linked with coronary heart disease (CHD). A high prevalence of genotypes $\varepsilon 4 /$ 3 and $\varepsilon 4 / 4$ has been meta-analytically associated with diagnosed CHD cases [1]. An association between apoE polymorphism and temperament was first found in a Young Finns cohort [2]. In a population-based sample of 1577 participants, motor activity in childhood and mental vitality, sociability and positive emotionality in adolescence were associated with the apoE polymorphism.

\footnotetext{
"Corresponding author.
}

Two research groups have attempted to replicate these findings. Tsai, Yu and Hong [3] found no associations between temperament and apoE. However, the study lacked statistical power due to a small sample that included a total of 135 subjects of whom only 32 were $\varepsilon 4$-carriers. Jorm and colleagues [4] examined activity in a sample of 681 participants and found no significant associations after controlling for multiple testing. The contradictory results may reflect differences in temperamental phenotypes, because different assessments were used in different studies. Jorm and colleagues [4] used, for instance, such methods in assessing activity and hyperactivity as 
the Rutter Problem Behavior Questionnaire [5], and Tsai and colleagues [3] used Cloninger's Tridimensional Personality Questionnaire [6]. Neither study used the same temperament dimensions as in our original study [2]. Furthermore, there might be temperamental differences between populations.

The purpose of this study is to confirm the original finding of the Young Finns Study in a sample that consists of the participants in the original article [2] supplemented with over 1200 new participants who were part of the same Young Finns Study, but not included in the original article. New and more reliable techniques in genotyping were used in the new sample, which remarkably reduce the risk of human error which might account for even as much as over $90 \%$ of the errors in genotyping [7]. Confirmation of the association between temperament and $a p o E$ is of importance because childhood temperament has been associated with somatic CHD risk factors in both childhood [8] and in adulthood [9,10] and $\varepsilon 4-$ variants of the $a p o E$ gene constitute a well-known risk for CHD [1,11]. There might be some shared biological basis underlying both CHD and temperament, and apoE might be one part of this shared background.

\section{Methods}

\subsection{Participants}

The participants were drawn from an ongoing population-based prospective Young Finns Study which began in 1980. The original sample at the baseline comprised 3596 participants from six age cohorts, 3, 6, 9, 12, 15 and 18-year-olds, with different geographical and socioeconomic backgrounds. An equal number of urban and rural boys and girls were randomly chosen from the national population register to ensure national representativeness of the Finns, as comprehensively detailed previously [12]. The study had the approval of the local ethics committees and the participants gave written informed consent.

All the 3596 participants were invited to participate in genotype and temperament assessments. ApoE genotypeing was conducted on 2946 persons who agreed to participate, of whom 2643 were genotyped by new methods and 303 had been genotyped earlier with an older method. The participants whose genotypes differed between the two measurement times were excluded from the analyses ( $n=27$ ) and thus apoE genotypes were available altogether on 2919 participants. Sufficient information on temperament was received from 2808 genotyped participants at the baseline in 1980 and from 2367 genotyped participants (52.6\% women, $47.4 \%$ men) at the first follow-up in 1983. All the participants who had been successfully genotyped and had sufficient temperament data on any of the assessed temperament scales comprised the present study sample. The participants were divided into two age groups: the three youngest age cohorts (3 - 9 years at the baseline and 6 - 12 years at the follow-up) were combined as a children's group. Similarly, the three oldest age cohorts (12 - 18 years at the baseline and 15 21 years at the follow-up) were combined as an adolescent's group.

\subsection{ApoE Genotyping}

New apoE genotyping was conducted by analyzing two SNPs (rs429358 and rs7412). Genomic DNA was extracted from peripheral blood leukocytes by using the QIAampÒDNA Blood Minikit and automated biorobot M48 extraction (Qiagen, Hilden, Germany). Genotyping was performed by using TaqmanÒSNP Genotyping Assays (rs429358 assay C 3084793_20; rs7412 assay C_904973_10) and the ABI Prism 7900HT Sequence Detection System (Applied Biosystems, Foster City, CA, USA). Discrepancies did not emerge in the genotyping results of duplicate samples. The old method for genotyping apoE was based on isoelectric focusing, cysteamine treatment and immunoblotting from delipidated plasma and has been documented in detail elsewhere [13]. The frequencies of the $a p o E$ genotypes in the present sample were 5, 173, 53, 1631, 849 and 97 in the genotype groups of $\varepsilon 2 / 2, \varepsilon 3 / 2, \varepsilon 4 / 2, \varepsilon 3 / 3, \varepsilon 4 / 3$ and $\varepsilon 4 / 4$, respectively. Group $\varepsilon 2 / 2$ was excluded from the analyses due to its small sample size $(n=5)$.

\subsection{Temperament}

The same methods in assessing temperament were used as in our original study [2]. First, mothers assessed the participants' temperament at the baseline in 1980 (participants aged 3 - 18) and at the follow-up in 1983 (participants aged 6 - 21) on scales derived from the Health Examination Survey [14]. Motor activity was evaluated on a four-point continuum: 1) always controlled; 2) overactive or restless only occasionally, for instance, when tired; 3) continuously more active than the average child or youth, and 4) always extremely active and energetic, even restless. Low cooperativeness was evaluated on a three-point continuum: 1) always very cooperative and responsive to others; 2) sometimes problems with peers, but mostly cooperative, and 3) continuous problems in cooperating with peers. Negative emotionality was assessed with eight items (e.g., “Other children's parents often complain about the child's behavior”) on 2-, 3- and 5-point Likert-type scales. Because of different scaling, standardized scores were used. A mean score (for negative emotionality) was computed if the participant's mother had answered at least $50 \%$ of the items. Reliabilities (Cronbach's alphas) for negative emotionality were 0.62 at the baseline and 0.80 at the follow-up.

Second, in 1983 mothers also assessed their children's temperament with the semantic differential measure that was based on the Operation Family Study [15]. The meas- 
ure consisted of seven items that each had two opposite attributes and were ranked on 5-point scales. Mental vitality was evaluated by three items (passive-active, listless-full of energy, unresponsive-alert); sociability was evaluated by two items (alone-sociable, quiet-talkative), and positive emotionality was evaluated by two items (depressed-happy, hostile-friendly). Sum scores were used to calculate each scale and due to the low number of items on the scales no missing answers were allowed. Reliabilities (Cronbach's alphas) in the present sample were 0.47 for mental vitality, 0.54 for sociability and 0.41 for positive emotionality.

In addition to mother-ratings, self-ratings on temperament were also made in 1983 by adolescent participants (aged 15 - 21) with the semantic differential measure. The scales mental vitality, sociability and positive emotionality were calculated similarly as described above. Reliabilities (Cronbach's alphas) in the present sample for self-rated temperament were 0.48 for mental vitality, 0.54 for sociability and 0.38 for positive emotionality.

\subsection{Statistical Methods}

Analyses were conducted separately for children and adolescents. The analysis of covariance was carried out between five genotype groups of apoE and each tem- perament dimension separately. First, all possible sex $\times$ apoE interactions predicting temperament were tested. As all interactions were non-significant, sexes were analyzed together with sex as a covariate.

\section{Results}

The means of the temperament dimensions of motor activity, cooperativeness and negative emotionality did not differ between the apoE groups. All associations were nonsignificant at the baseline and at the follow-up among both children and adolescents (all p-values $>0.10$ ). This replicates our original results on absence of association of $a p o E$ with cooperativeness, negative emotionality and adolescents' motor activity. The original significant finding ( $p<0.05$ ) of children's motor activity did not replicate, as children's motor activity was now unrelated to the apoE polymorphisms ( $\mathrm{p}=0.145)$.

Table 1 presents the means of temperament dimensions of mental vitality, sociability and positive emotionality. Children's temperament was unrelated to the apoE polymorphisms (all p-values $>0.60$ ) replicating our previous finding. In adolescence, mental vitality, $\mathrm{F}(4,983)=$ 5.05, $\mathrm{p}<0.001$, sociability, $\mathrm{F}(4,1002)=4.02, \mathrm{p}=0.003$, and positive emotionality, $\mathrm{F}(4,996)=3.76, \mathrm{p}=0.005$,

Table 1. Sex-adjusted temperament means and sex-adjusted differences between apoE genotypes. The original 1993 reported means and significances are shown below [2].

\begin{tabular}{|c|c|c|c|c|c|c|c|c|c|c|c|c|}
\hline & Age & $\mathbf{n}$ & $\varepsilon 3 / 2$ & $\mathbf{n}$ & $\varepsilon 4 / 2$ & $\mathbf{n}$ & $\varepsilon 3 / 3$ & $\mathbf{n}$ & $\varepsilon 4 / 3$ & $\mathbf{n}$ & $\varepsilon 4 / 4$ & p-Value \\
\hline \multicolumn{13}{|l|}{ Mothers' assessment } \\
\hline \multicolumn{13}{|l|}{ Mental vitality } \\
\hline & $6-12$ & 90 & 11.2 & 20 & 11.6 & 740 & 11.3 & 394 & 11.3 & 49 & 11.3 & ns \\
\hline \multirow[t]{2}{*}{1993 reported: } & $6-12$ & & 11.0 & & 11.7 & & 11.4 & & 11.4 & & 11.2 & ns \\
\hline & $15-21$ & 56 & $10.3^{\mathrm{abc}}$ & 25 & $10.1^{\text {def }}$ & 574 & $11.1^{\text {ad }}$ & 305 & $11.2^{\text {be }}$ & 29 & $11.7^{\mathrm{cf}}$ & $<0.001$ \\
\hline 1993 reported: & $15-21$ & & 10.1 & & 9.2 & & 11.0 & & 11.4 & & 12.0 & $<0.001$ \\
\hline \multicolumn{13}{|l|}{ Sociability } \\
\hline & $6-12$ & 92 & 7.6 & 20 & 7.6 & 757 & 7.7 & 403 & 7.7 & 50 & 7.5 & ns \\
\hline \multirow[t]{2}{*}{1993 reported: } & $6-12$ & & 7.7 & & 8.1 & & 7.7 & & 7.8 & & 7.4 & ns \\
\hline & $15-21$ & 56 & $7.1^{\mathrm{a}}$ & 25 & $6.5^{\mathrm{bc}}$ & 587 & $7.3^{\text {bd }}$ & 310 & $7.6^{\text {acde }}$ & 30 & $6.9^{\mathrm{e}}$ & $<0.01$ \\
\hline 1993 reported: & $15-21$ & & 6.9 & & 5.8 & & 7.3 & & 7.5 & & 7.4 & $<0.01$ \\
\hline \multicolumn{13}{|l|}{ Positive emotionality } \\
\hline \multirow{2}{*}{1993 reported: } & $6-12$ & 92 & 7.9 & 20 & 8.0 & 744 & 7.9 & 397 & 8.0 & 50 & 7.8 & ns \\
\hline & $15-21$ & 56 & $7.7^{\mathrm{a}}$ & 24 & $7.3^{\mathrm{bc}}$ & 582 & $7.7^{\mathrm{d}}$ & 310 & $7.9^{\text {be }}$ & 30 & $8.5^{\text {acde }}$ & $<0.01$ \\
\hline 1993 reported: & $15-21$ & & 7.3 & & 7.5 & & 7.7 & & 7.9 & & 8.8 & $<0.01$ \\
\hline \multicolumn{13}{|l|}{ Self-assessment } \\
\hline \multicolumn{13}{|l|}{ Mental vitality } \\
\hline & $15-21$ & 57 & $10.2^{\mathrm{a}}$ & 25 & 10.2 & 621 & $10.5^{\mathrm{b}}$ & 325 & $10.8^{\mathrm{ab}}$ & 31 & 10.8 & 0.05 \\
\hline 1993 reported: & $15-21$ & & 9.9 & & 9.8 & & 10.4 & & 10.9 & & 11.0 & $<0.01$ \\
\hline \multicolumn{13}{|l|}{ Sociability } \\
\hline & $15-21$ & 57 & 6.7 & 25 & 6.7 & 625 & 7.2 & 323 & 7.2 & 31 & 7.0 & ns \\
\hline 1993 reported: & $15-21$ & & 6.5 & & 6.8 & & 7.2 & & 7.2 & & 7.3 & ns \\
\hline \multicolumn{13}{|l|}{ Positive emotionality } \\
\hline & $15-21$ & 57 & 7.5 & 24 & $7.2^{\mathrm{a}}$ & 628 & $7.5^{\mathrm{bc}}$ & 325 & $7.7^{\mathrm{b}}$ & 30 & $7.9^{\mathrm{ac}}$ & $<0.01$ \\
\hline 1993 reported: & $15-21$ & & 7.1 & & 7.3 & & 7.4 & & 7.7 & & 8.1 & $<0.01$ \\
\hline
\end{tabular}

${ }^{\text {a-f }}$ Pair-wise comparisons, groups that are marked with the same letter, differ statistically significantly from each other (p < 0.05); Note: The 1993 reported means and differences were first published in Psychosomatic Medicine, Vol. 55, No. 2, 1993, pp. 155-163. 
were all associated with apoE polymorphisms when assessed by mothers. Similarly as in our previous study, the highest mental vitality and positive emotionality were found in the $\varepsilon 4 / 4$ group, whereas the highest sociability was found in the $\varepsilon 4 / 3$ group. Thus, the results on mental vitality, sociability and positive emotionality assessed by mothers replicate our original findings. When temperament was self-rated by adolescents, positive emotionality, $\mathrm{F}(4,1058)=3.44, \mathrm{p}=0.008$, differed between the apoE groups. The association between mental vitality and $a p o E$, which was significant in our previous study ( $p<0.01$ ), was only of borderline significance, $F(4,1053)=2.35, p$ $=0.052$. As in our previous study, the highest positive emotionality was found in the $\varepsilon 4 / 4$ group and the highest mental vitality in the $\varepsilon 4 / 3$ and $\varepsilon 4 / 4$ groups, although the means of mental vitality did not differ significantly in the pair-wise comparisons for $\varepsilon 4 / 4$. Furthermore, as previously, sociability was not associated with the $a p o E$ polymorphisms ( $p=0.095)$. Thus, only the results on mental vitality differed from our original findings conducted with self-ratings.

\section{Discussion}

The present findings confirm an association between temperament and $a p o E$ in a larger and more reliably genotyped sample, using the same temperament dimensions as in our original study [2]. Adolescents scoring high on mental vitality, positive emotionality and sociability carried $\varepsilon 4 / 4$ or $\varepsilon 4 / 3$ variants of the apoE gene. The weakest association of our original article [2], that between motor activity and apoE, was no longer found. In accordance with our previous findings, associations were found in adolescents, but not children, which might reflect instability of personality in childhood compared to adolescence or adulthood [16].

The studies mentioned in the introduction [3,4] did not replicate our original findings [2]. The contradictory findings might result from differences in temperament assessments leading to different phenotypes. All the abovementioned studies used measures different from our original study; therefore, they may have examined slightly different phenomena.

A great variance of phenotypes belongs to the core problems of behavioral genetics. In spite of high expectancy the studies of behavioral genetics have not provided satisfactory results in personality psychology. This is, at least partly, caused by a wide collection of measures used in different studies. Perhaps more importantly, even the same measures do not assess the same personality traits in different cultures.

The original finding was from a study among Finns [2] while the other studies were conducted in Australian [4] and Chinese [3] cultures. Cross-cultural personality differences have been found. For example, Finns report more temperamental positive affectivity [17] and less reward dependence and persistence [18] than Americans. Cross-cultural differences may reflect real personality distinctions between cultures that are due to differences in child-rearing practices, attitudes, values and expectations. In addition, the same personality traits are variously perceived, valued and accepted in different cultures. Reference-group effect is one regulating factor of personality self-assessments. People tend to assess themselves compared to others and the others to whom they compare themselves may differ from population to population $[19,20]$. Thus, cross-cultural personality differences might reflect true personality distinctions or they may reflect differences in culture-related attitudes and values, even culture-related response styles.

When a genetic background of a personality trait is found in one culture, replicating the results requires finding a proper measure that captures the same personality trait phenotype and phenomenon in another culture. It has been meta-analytically shown that the trait dimensions that are thought to reflect similar latent traits are not all associated with same gene polymorphisms in different cultures [21]. Furthermore, in a genome-wide association study that used exactly the same methods in assessing temperament, weak support for similar genetic associations between Finnish and Australian populations was found for only some of the traits [22], implying that some trait phenotypes are more culture-specific than others. Thus, it seems likely that the discrepancies between temperament and apoE findings might be affected by cultural differences.

In addition to cultural differences in personality assessment, there are also biological differences between populations. Finns have an overrepresentation of the $a p o E$ $\varepsilon 4$-allele [13] and a larger representation of an allele increases power to detect phenotypic differences related to different genotypes [23]. Thus, it is possible that the previous replication studies $[3,4]$ lacked statistical power to detect differences between the different genotypes and the temperamental phenotypes. Our larger representation of the $\varepsilon 4$-allele might enable us to detect quite small but still significant temperament differences between different apoE groups.

It has been shown that $\varepsilon 4 / 3$ and $\varepsilon 4 / 4$ variants of the apoE gene constitute an evident risk for CHD [1,11]. In our studies, high mental vitality, positive emotionality and sociability-i.e., a tendency to enjoy another's company-were associated with the risk genotypes of the apoE gene. At first, this may seem contradictory, because all those traits are considered "positive" characteristics and rather socially protective than harmful personally traits. Sociability is especially highly appreciated in the Western cultures and has several positive outcomes $[24,25]$. However, we have previously shown that high 
sociability might be linked with an increased risk for CHD. For instance, high sociability has been associated with high serum insulin and low high-density lipoprotein cholesterol levels in childhood, adolescence and young adulthood $[8,26]$ and with high intima-media thickness in adulthood [27]. Supporting our findings, high sociability has also been associated with a higher risk for mental stress-induced ischemia among cardiac patients [28]. It might be that high sociability becomes a stressful characteristic when it leads to excessive eagerness to help and please others and to overachieve at work. Thus, function and health outcomes of sociability need further examination.

Other temperament dimensions that were now associated with the CHD risk genotypes of apoE, that is, high mental vitality and high positive emotionality, have previously been associated with low somatic risk factors of CHD in childhood and adolescence [8]. This suggests that genetic and behavioral risk factors may function in different directions, i.e., temperament might be genetically associated with CHD risk, but at the same time protect from illness through tendencies in behavior. The present study confirms our previous finding that there is an association between temperament and apoE [2]. Temperament characteristics might contribute to the pathogenesis of CHD, even though their specific roles need further examination.

\section{Acknowledgements}

The Young Finns Study has been financially supported by the Academy of Finland projects 258578 (M. H.), 258711 (L. K.-J.), 126925 (O. T. R.), 121584 (O. T. R.), 124282 (O. T. R.), 129378 (O. T. R), 117797 (O. T. R.), 41071 (O. T. R.), 129398 (T. L.) and 117941 (T. L.), and grants from the Emil Aaltonen Foundation (M. H., O. T. R., T. L.), the Ella \& Georg Ehrnrooth Foundation (M. H.), the Signe \& Ane Gyllenberg Foundation (M. H., L. K.-J.), the Research Funds of the University of Helsinki (M. H.), the Finnish Cultural Foundation (P. M., O. T. R.), the Alfred Kordelin Foundation (P. M.), the Social Insurance Institution of Finland (O. T. R.), Kuopio, Tampere and Turku University Hospital Medical Funds (O. T. R.), the Juho Vainio Foundation (O. T. R.), the Paavo Nurmi Foundation (O. T. R.), the Finnish Foundation of Cardiovascular Research (O. T. R., T. L.), the Sigrid Juselius Foundation (O. T. R.), the Tampere Tuberculosis Foundation (T. L.) and the Competitive Research Funding of Tampere University Hospital (T. L.). The expert technical assistance in data management by Irina Lisinen and Ville Aalto are gratefully acknowledged.

\section{REFERENCES}

[1] Y. Song, M. J. Stampfer and S. Liu, "Meta-Analysis: Apolipoprotein E Genotypes and Risk for Coronary Heart
Disease," Annals of Internal Medicine, Vol. 141, No. 2, 2004, pp. 137-147.

doi:10.7326/0003-4819-141-2-200407200-00013

[2] L. Keltikangas-Järvinen, K. Räikkönen and T. Lehtimäki, "Dependence between Apolipoprotein E Phenotypes and Temperament in Children, Adolescents, and Young Adults," Psychosomatic Medicine, Vol. 55, No. 2, 1993, pp. 155163.

[3] S. J. Tsai, Y. W. Yu and C. J. Hong, "Personality Traits in Young Female Apolipoprotein E (apoE) Epsilon4 and Non-Epsilon4 Carriers," American Journal of Medical Genetics. Part B, Neuropsychiatric Genetics, Vol. 124, No. 1, 2004, pp. 58-60. doi:10.1002/ajmg.b.20052

[4] A. F. Jorm, M. Prior, A. Sanson, D. Smart, Y. Zhang, et al., "Apolipoprotein E Genotype and Temperament: A Longitudinal Study from Infancy to the Late Teens,” Psychosomatic Medicine, Vol. 65, No. 4, 2003, pp. 662-664. doi:10.1097/01.PSY.0000077506.29218.60

[5] M. Rutter, J. Tizard and K. Whitmore, "Education, Health and Behaviour,” Longman, London, 1970.

[6] C. R. Cloninger, T. R. Przybeck and D. M. Svrakic, "The Tridimensional Personality Questionnaire: US Normative Data,” Psychological Reports, Vol. 69, No. 1, 1991, pp. 1047-1057.

[7] F. Pompanon, A. Bonin, E. Bellemain and P. Taberlet, "Genotyping Errors: Causes, Consequences and Solutions," Nature Reviews Genetics, Vol. 6, No. 11, 2005, pp. 847859. doi: $10.1038 / \mathrm{nrg} 1707$

[8] N. Ravaja and L. Keltikangas-Järvinen, “Temperament and Metabolic Syndrome Precursors in Children: A ThreeYear Follow-Up,” Preventive Medicine, Vol. 24, No. 5, 1995, pp. 518-527. doi:10.1006/pmed.1995.1082

[9] L. Keltikangas-Järvinen, L. Pulkki-Råback, S. Puttonen, J. Viikari and O. T. Raitakari, "Childhood Hyperactivity as a Predictor of Carotid Artery Intima Media Thickness over a Period of 21 Years: The Cardiovascular Risk in Young Finns Study," Psychosomatic Medicine, Vol. 68, No. 4, 2006, pp. 509-516.

doi:10.1097/01.psy.0000227752.24292.3e

[10] L. Pulkki-Råback, M. Elovainio, M. Kivimäki, O. T. Raitakari and L. Keltikangas-Järvinen, "Temperament in Childhood Predicts Body Mass in Adulthood: The Cardiovascular Risk in Young Finns Study,” Health Psychology, Vol. 24, No. 3, 2005, pp. 307-315. doi:10.1037/0278-6133.24.3.307

[11] A. M. Bennet, E. Di Angelantonio, Z. Ye, F. Wensley, A. Dahlin, et al., "Association of Apolipoprotein E Genotypes with Lipid Levels and Coronary Risk," Journal of the American Medical Association, Vol. 298, No. 11, 2007, pp. 1300-1311. doi:10.1001/jama.298.11.1300

[12] H. K. Åkerblom, M. Uhari, E. Pesonen, M. Dahl, E. A. Kaprio, et al., "Cardiovascular Risk in Young Finns," Annals of Medicine, Vol. 23, No. 1, 1991, pp. 35-39. doi:10.3109/07853899109147928

[13] T. Lehtimäki, T. Moilanen, J. Viikari, H. K. Åkerblom, C. Ehnholm, et al., "Apolipoprotein E Phenotypes in Finnish Youths: A Cross-Sectional and 6-Year Follow-Up Study," Journal of Lipid Research, Vol. 31, No. 3, 1990, pp. 487495. 
[14] J. Roberts and J. T. Baird, "Behavior Patterns of Children in School," Vital and Health Statistics, Vol. 11, No. 113, 1972.

[15] T. Makkonen, I. Ruoppila, T. Rönkä, S. Timonen, L. Valvanne, et al., "Operaatio Perhe-Isä ja Synnytys [“Operation Family” (Child Report no. A34)],” Mannerheim League of Child Welfare, Helsinki, Finland, 1981.

[16] A. Caspi, B. W. Roberts and R. L. Shiner, "Personality Development: Stability and Change," Annual Review of Psychology, Vol. 56, 2005, pp. 453-484. doi:10.1146/annurev.psych.55.090902.141913

[17] L. M. Gaias, K. Räikkönen, N. Komsi, M. A. Gartstein, P. A. Fisher, et al., "Cross-Cultural Temperamental Differences in Infants, Children, and Adults in the United States of America and Finland," Scandinavian Journal of Psychology, Vol. 53, No. 2, 2012, pp. 119-128. doi:10.1111/j.1467-9450.2012.00937.x

[18] J. Miettunen, L. Kantojärvi, J. Veijola, M. R. Järvelin and M. Joukamaa, "International Comparison of Cloninger's Temperament Dimensions,” Personality and Individual Differences, Vol. 41, No. 8, 2006, pp. 1515-1526. doi:10.1016/i.paid.2006.06.006

[19] S. J. Heine, D. R. Lehman, K. Peng and J. Greenholtz, "What's Wrong with Cross-Cultural Comparisons of Subjective Likert Scales? The Reference-Group Effect," Journal of Personality and Social Psychology, Vol. 82, No. 6, 2002, pp. 903-918. doi:10.1037/0022-3514.82.6.903

[20] M. Ross, S. J. Heine, A. E. Wilson and S. Sugimori, "CrossCultural Discrepancies in Self-Appraisals,” Personality and Social Psychology Bulletin, Vol. 31, No. 9, 2005, pp. 1175-1188. doi: $10.1177 / 0146167204274080$

[21] M. R. Munafo, B. Yalcin, S. A. Willis-Owen and J. Flint, "Association of the Dopamine D4 Receptor (DRD4) Gene and Approach-Related Personality Traits: MetaAnalysis and New Data,” Biological Psychiatry, Vol. 63, No. 2, 2008, pp. 197-206. doi:10.1016/j.biopsych.2007.04.006

[22] S. K. Service, K. J. H. Verweij, J. Lahti, E. Congdon, J. Ekelund, et al., "A Genome-Wide Meta-Analysis of Association Studies of Cloninger's Temperament Scales,"
Translational Psychiatry, Vol. 2, No. e116, 2012. doi:10.1038/tp.2012.37

[23] A. Caspi, A. R. Hariri, A. Holmes, R. Uher and T. E. Moffitt, "Genetic Sensitivity to the Environment: The Case of the Serotonin Transporter Gene and its Implications for Studying Complex Diseases and Traits," American Journal of Psychiatry, Vol. 167, No. 5, 2010, pp. 509-527. doi:10.1176/appi.ajp.2010.09101452

[24] M. Hintsanen, T. Hintsa, A. Widell, M. Kivimäki, O. T. Raitakari, et al., "Negative Emotionality, Activity, and Sociability Temperaments Predicting Long-Term Job Strain and Effort-Reward Imbalance: A 15-Year Prospective Follow-Up Study," Journal of Psychosomatic Research, Vol. 71, No. 2, 2011, pp. 90-96. doi:10.1016/j.jpsychores.2011.02.012

[25] A. D. Clemets and B. A. Bailey, "The Relationship between Temperament and Anxiety: Phase I in the Development of a Risk Screening Model to Predict Stress-Related Health Problems,” Journal of Health Psychology, Vol. 15, No. 4, 2010, pp. 515-525. doi:10.1177/1359105309355340

[26] L. Keltikangas-Järvinen, N. Ravaja and J. Viikari, "Identifying Cloninger's Temperament Profiles as Related to the Early Development of the Metabolic Cardiovascular Syndrome in Young Men," Arteriosclerosis, Thrombosis \& Vascular Biology, Vol. 19, No. 8, 1999, pp. 1998-2006. doi:10.1161/01.ATV.19.8.1998

[27] M. Hintsanen, L. Pulkki-Råback, M. Juonala, J. S. A. Viikari, O. T. Raitakari, et al., "Cloninger’s Temperament Traits and Preclinical Atherosclerosis: The Cardiovascular Risk in Young Finns Study," Journal of Psychosomatic Research, Vol. 67, No. 1, 2009, pp. 77-84. doi:10.1016/j.jpsychores.2009.01.002

[28] M. W. Ketterer, K. E. Freedland, D. S. Krantz, P. Kaufmann, S. Forman, et al., "Psychological Correlates of Mental Stress-Induced Ischemia in the Laboratory: The Psychophysiological Investigation of Myocardial Ischemia (PIMI) Study," Journal of Health Psychology, Vol. 5, No. 1, 2000, pp. 75-85. doi:10.1177/135910530000500112 